\title{
Interval-Set Algebra for Qualitative Knowledge Representation
}

\author{
Y.Y. Yao \\ Department of Mathematical Sciences, Lakehead University \\ Thunder Bay, Ontario, Canada P7B 5E1
}

\begin{abstract}
The notion of interval sets is introduced as a new kind of sets, represented by a pair of sets, namely, the lower and upper bounds. The interval-set algebra may be regarded as a counterpart of the interval-number algebra. It provides a useful tool to represent qualitative information. Operations on interval sets are also defined, based on the corresponding set-theoretic operations on their members. In addition, basic properties of interval-set algebra are examined, and the relationships between interval sets, rough sets and fuzzy sets are analyzed.
\end{abstract}

\section{Introduction}

The contemporary concern about knowledge representation and reasoning with uncertain information has generated many extensions of classical mathematical tools. One class of these extensions is the use of lower and upper bounds of the true value, and the development of the corresponding mechanism for processing such interval representations [7]. For example, in the Dempster-Shafer theory [9], both a lower and an upper bound are maintained regarding the uncertainty of a proposition. The lower bound is characterized by a belief function which represents the belief one actually commits to the proposition. The upper bound is defined by a plausibility function which represents the maximum belief one may commit to the proposition. A pair of belief and plausibility functions may be interpreted as bounds of a family of probability functions. Given this interpretation, belief and plausibility functions may be considered as extensions of probability functions.

Motivated by the need for error analysis in numeric computation, Moore [5] proposed an interval-number algebra by extending elementary arithmetic to interval arithmetic. An interval number is defined by its lower and upper bounds. Arithmetic operations on interval numbers are carried out based on arithmetic opera- tions on real numbers. The use of interval numbers enables us to estimate the bounds for errors caused by a computer's incapability to represent precisely a real number. Recently, a number of extensions to intervalnumber algebra have been reported by Parsons [7], in which a closed interval of an ordered set is used instead of a closed interval of real numbers.

The notion of rough sets advocated by Pawlak [8] provides another interesting research topic in interval analysis. In this framework, a set is approximated by a pair of lower and upper approximations. The lower approximation consists of elements that definitely belong to the set, whereas the upper approximation consists of elements that possibly belong to the set. In other words, rough sets are bounds of an ordinary set. Therefore, rough sets are used to approximate ordinary sets in the same way that interval numbers are used to approximate real numbers in interval-number algebra. Farinas del Cerro and Prade [4] suggested that it is useful to regard the lower and upper approximations as a pair and to define operations on such pairs. Similar ideas were also discussed in the study of flou sets [6].

The main objective of this paper is to introduce an interval-set algebra, the counterpart of intervalnumber algebra, for representing qualitative information. Interval-number algebra will be briefly reviewed to establish the ground work of the study. The notion of interval sets is defined as a natural consequence of our inability to characterize a set precisely. The basic properties of interval sets are identified according to their physical interpretations. Operations on interval sets are defined in a manner parallel to that used in defining operations on interval numbers. More justifications for the proposed interval-set algebra are provided by demonstrating its connections with rough sets and fuzzy sets. Although the proposed intervalset algebra is similar to the notion of flou sets [6], the analysis of the relationships between interval-set algebra, interval-number algebra, rough sets and fuzzy sets is very important for qualitative knowledge representation using interval sets. 


\section{Interval-Number Algebra}

For completeness, this section briefly reviews the interval-number algebra proposed by Moore [1, 5].

An interval number is an ordered pair of real numbers, $\left[a_{1}, a_{2}\right]$, with $a_{1} \leq a_{2}$. It is also a set of real numbers defined by:

$$
\left[a_{1}, a_{2}\right]=\left\{x \mid a_{1} \leq x \leq a_{2}\right\}
$$

The set of all interval numbers is denoted by $I(\Re)$. Degenerate intervals of the form $[a, a]$ are equivalent to real numbers. Obviously, the notion of interval numbers provides a tool for representing a real number by specifying its lower and upper endpoints. This representation scheme is very useful in situations where we are unable to obtain a precise measurement of a physical quantity (i.e., inexact experimental measurement), or where we cannot store a real number with sufficient precision in a computer due to space limitation (i.e., insufficient representation).

We can perform arithmetic with interval numbers (i.e., sets of real numbers) through the arithmetic operations on their members. Let $I$ and $J$ be two interval numbers, and let $*$ denote an arithmetic operation + , ,$- \cdot$ or / on pairs of real numbers. Then an arithmetic operation $*$ may be extended to pairs of interval numbers $I, J$ by

$$
I * J=\{x * y \mid x \in I, y \in J\} .
$$

The result $I * J$ is again a closed bounded interval unless $0 \in J$ and the operation $*$ is division (in which case, $I * J$ is undefined). In fact, we can derive the following formulas for $I * J$ :

$$
\begin{aligned}
& {\left[a_{1}, a_{2}\right]+\left[b_{1}, b_{2}\right]=\left[a_{1}+b_{1}, a_{2}+b_{2}\right],} \\
& {\left[a_{1}, a_{2}\right]-\left[b_{1}, b_{2}\right]=\left[a_{1}-b_{2}, a_{2}-b_{1}\right] \text {, }} \\
& {\left[a_{1}, a_{2}\right] \cdot\left[b_{1}, b_{2}\right]=\left[\min \left(a_{1} b_{1}, a_{1} b_{2}, a_{2} b_{1}, a_{2} b_{2}\right),\right.} \\
& \left.\max \left(a_{1} b_{1}, a_{1} b_{2}, a_{2} b_{1}, a_{2} b_{2}\right)\right], \\
& {\left[a_{1}, a_{2}\right] /\left[b_{1}, b_{2}\right]=\left[a_{1}, a_{2}\right] \cdot\left[1 / b_{2}, 1 / b_{1}\right] \text { for }} \\
& 0 \notin\left[b_{1}, b_{2}\right] \text {. }
\end{aligned}
$$

The arithmetic system defined above is called interval arithmetic or interval-number algebra. Many properties of the arithmetic operations on pairs of real numbers can be carried over to the new arithmetic operations on pairs of interval numbers. For example, the addition operation + on interval numbers is also associative and commutative. A more complete list of such properties can be found in Moore [5], and Alefeld and Herzberger [1].

\section{Interval-Set Algebra}

This section introduces the notion of interval sets in parallel to that of interval numbers. The operations on interval sets are defined as extensions of the ordinary set-theoretic operations on their members.

Let $U$ be a finite set, called the universe or the reference set, and $2^{U}$ be its power set. A subset of $2^{U}$ of the form

$$
\mathcal{A}=\left[A_{1}, A_{2}\right]=\left\{A \in 2^{U} \mid A_{1} \subseteq A \subseteq A_{2}\right\}
$$

is called a closed interval set, where it is assumed $A_{1} \subseteq$ $A_{2}$. The set of all closed interval sets is denoted by $I\left(2^{U}\right)$. Degenerate interval sets of the form $[A, A]$ are equivalent to ordinary sets. Thus, interval sets may be considered as an extension of elementary sets.

Let $\cap, \cup$ and - be the usual set intersection, union and difference defined on $2^{U}$, respectively. We define the following binary operations on $I\left(2^{U}\right)$ : for two interval sets $\mathcal{A}=\left[A_{1}, A_{2}\right]$ and $\mathcal{B}=\left[B_{1}, B_{2}\right]$,

$$
\begin{aligned}
& \mathcal{A} \sqcap \mathcal{B}=\{A \cap B \mid A \in \mathcal{A}, B \in \mathcal{B}\}, \\
& \mathcal{A} \sqcup \mathcal{B}=\{A \cup B \mid A \in \mathcal{A}, B \in \mathcal{B}\}, \\
& \mathcal{A} \backslash \mathcal{B}=\{A-B \mid A \in \mathcal{A}, B \in \mathcal{B}\} .
\end{aligned}
$$

These operations are referred to as interval-set intersection, union and difference. They are closed on $I\left(2^{U}\right)$, namely, $\mathcal{A} \sqcap \mathcal{B}, \mathcal{A} \sqcup \mathcal{B}$ and $\mathcal{A} \backslash \mathcal{B}$ are interval sets. In fact, these interval sets can be explicitly computed by using the following formulas:

$$
\begin{aligned}
\mathcal{A} \sqcap \mathcal{B} & =\left[A_{1} \cap B_{1}, A_{2} \cap B_{2}\right], \\
\mathcal{A} \sqcup \mathcal{B} & =\left[A_{1} \cup B_{1}, A_{2} \cup B_{2}\right], \\
\mathcal{A} \backslash \mathcal{B} & =\left[A_{1}-B_{2}, A_{2}-B_{1}\right] .
\end{aligned}
$$

Similarly, the interval-set complement $\neg\left[A_{1}, A_{2}\right]$ of $\left[A_{1}, A_{2}\right]$ is defined as $[U, U] \backslash\left[A_{1}, A_{2}\right]$. This is equivalent to $\left[U-A_{2}, U-A_{1}\right]=\left[A_{2}^{c}, A_{1}^{c}\right]$, where $A^{c}=U-A$ denote the usual set complement operation. Clearly, we have $\neg[\emptyset, \emptyset]=[U, U]$ and $\neg[U, U]=[\emptyset, \emptyset]$.

With the above definition operations $\sqcap, \sqcup$ and $\neg$, $I\left(2^{U}\right)$ is a completely distributive lattice [6]. Both $\sqcap$ and $\sqcup$ are idempotent, commutative, associative, absorptive and distributive. For interval-set complement, De Morgan's laws and Double negation law hold. Moreover, $[U, U]$ and $[\emptyset, \emptyset]$ are the unique identities for interval-set intersection and union. These properties may be regarded as the counterparts of the properties of their corresponding set-theoretic operations. Unlike elementary set theory, for an interval set $\mathcal{A}, \mathcal{A} \sqcap \neg \mathcal{A}$ is not necessarily equal to $[\emptyset, \emptyset]$, and $\mathcal{A} \sqcup \neg \mathcal{A}$ is not necessarily equal to $[U, U]$. In addition, 
$\mathcal{A} \backslash \mathcal{A}$ is not necessarily equal to $[\emptyset, \emptyset]$. Nevertheless, $\emptyset \in \mathcal{A} \sqcap \neg \mathcal{A}, U \in \mathcal{A} \sqcup \neg \mathcal{A}$, and $\emptyset \in \mathcal{A} \backslash \mathcal{A}$.

Interval sets are subsets of the power set $2^{U}$. The symbols $\in, \subseteq,=, \cap, \cup$ may be used, in their usual settheoretic sense, to represent relationships between elements of $2^{U}$ and an interval set, and between different interval sets. Thus, $A \in\left[A_{1}, A_{2}\right]$ means that $A$ is a subset of $U$ such that $A_{1} \subseteq A \subseteq A_{2}$. We write $\left[A_{1}, A_{2}\right] \subseteq\left[B_{1}, B_{2}\right]$ if the interval set $\left[A_{1}, A_{2}\right]$ as an ordinary set is contained in $\left[B_{1}, B_{2}\right]$ as an ordinary set. In other words, by $\left[A_{1}, A_{2}\right] \subseteq\left[B_{1}, B_{2}\right]$ we mean that $B_{1} \subseteq A_{1} \subseteq A_{2} \subseteq B_{2}$. Similarly, two interval sets are equal, written $\mathcal{A}=\mathcal{B}$, if they are equal in settheoretic sense, that is $\mathcal{A}=\mathcal{B}$ if and only if $A_{1}=B_{1}$ and $A_{2}=B_{2}$.

The proposed operations $\sqcap$, $\sqcup, \backslash$, and $\neg$ are not the same as the usual set-theoretic operations. The following relationship holds between the ordinary set and the interval-set intersections:

$$
\left[A_{1}, A_{2}\right] \cap\left[B_{1}, B_{2}\right] \subseteq\left[A_{1}, A_{2}\right] \sqcap\left[B_{1}, B_{2}\right] .
$$

However, there do not exist similar relationships between other set and interval-set operations. When only degenerate interval sets are used, the interval-set operations $\sqcap, \sqcup$ and $\backslash$ reduce to the usual set intersection, union and difference.

The inclusion of interval sets may be defined by:

$$
\left[A_{1}, A_{2}\right] \sqsubseteq\left[B_{1}, B_{2}\right] \Longleftrightarrow\left(A_{1} \subseteq B_{1}, A_{2} \subseteq B_{2}\right) .
$$

Based on this definition, for two interval sets $\mathcal{A}$ and $\mathcal{B}, \mathcal{A}=\mathcal{B}$ if and only if $\mathcal{A} \sqsubseteq \mathcal{B}$ and $\mathcal{B} \sqsubseteq \mathcal{A}$. For $\mathcal{A}, \mathcal{B}, \mathcal{C}, \mathcal{D} \in I\left(2^{U}\right)$, the following properties hold for the $\sqsubseteq$ relation:

$$
\begin{aligned}
& \mathcal{A} \sqsubseteq \mathcal{B} \Longleftrightarrow \mathcal{A} \sqcap \mathcal{B}=\mathcal{A}, \\
& \mathcal{A} \sqsubseteq \mathcal{B} \Longleftrightarrow \mathcal{A} \sqcup \mathcal{B}=\mathcal{B} ; \\
& \mathcal{A} \sqsubseteq \mathcal{B} \text { and } \mathcal{C} \sqsubseteq \mathcal{D} \Longrightarrow \mathcal{A} \sqcap \mathcal{C} \sqsubseteq \mathcal{B} \sqcap \mathcal{D}, \\
& \mathcal{A} \sqsubseteq \mathcal{B} \text { and } \mathcal{C} \sqsubseteq \mathcal{D} \Longrightarrow \mathcal{A} \sqcup \mathcal{C} \sqsubseteq \mathcal{B} \sqcup \mathcal{D} ; \\
& \mathcal{A} \sqcap \mathcal{B} \sqsubseteq \mathcal{A}, \quad \mathcal{A} \sqcap \mathcal{B} \sqsubseteq \mathcal{B}, \\
& \mathcal{A} \sqsubseteq \mathcal{A} \sqcup \mathcal{B}, \quad \mathcal{B} \sqsubseteq \mathcal{A} \sqcup \mathcal{B} .
\end{aligned}
$$

Like its counterpart in elementary set theory, the relation $\sqsubseteq$ on $I\left(2^{U}\right)$ is a reflexive and transitive relation. On the other hand, for two interval sets $\mathcal{A}$ and $\mathcal{B}$ with $\mathcal{A} \sqsubseteq \mathcal{B}$, the difference $\mathcal{A} \backslash \mathcal{B}$ is not necessarily equal to $[\emptyset, \bar{\emptyset}]$. In this case, we only have $\mathcal{A} \sqsubseteq \mathcal{B} \Longrightarrow \emptyset \in \mathcal{A} \backslash \mathcal{B}$.

In contrast to interval-number algebra, interval-set algebra puts forward a useful mechanism for representing uncertain qualitative information. An immediate application of interval-set algebra is qualitative reasoning in artificial intelligence. Bundy [2] provided a good example of such qualitative reasoning mechanisms in incidence calculus.

\section{Interpretations of Interval Sets}

This section compares the proposed interval-set algebra with rough sets and fuzzy sets, two complementary extensions of ordinary set theory [3]. The connections between these theories suggest methods for constructing interval sets, provide plausible interpretations of interval sets, and give further support to interval-set algebra.

\subsection{Rough sets}

The notion of rough sets was proposed by Pawlak [8] to describe an ordinary set by its lower and upper approximations.

Let $R_{E} \subseteq U \times U$ be an equivalence (indiscernibility) relation on the universe $U$, i.e., $R_{E}$ is reflexive, symmetric and transitive. The pair $A p r=\left(U, R_{E}\right)$ is called an approximation space. The equivalence relation $R_{E}$ partitions the set $U$ into disjoint subsets. Such a partition of the universe is denoted by $U / R_{E}=\left\{E_{1}, E_{2}, \ldots, E_{n}\right\}$, where $E_{i}$ is an equivalence class of $R_{E}$. If two elements $a, b$ in $U$ belong to the same equivalence class $E \in U / R_{E}$, we say that $a$ and $b$ are indistinguishable.

Given a subset $A \subseteq U$, one can describe it using a pair of lower and upper approximations induced by the equivalence classes of $U / R_{E}$ as follows:

$$
\begin{aligned}
& \underline{\operatorname{Apr}}(A)=\bigcup_{E_{i} \subseteq A} E_{i}, \\
& \overline{\operatorname{Apr}}(A)=\bigcup_{E_{i} \cap A \neq \emptyset} E_{i} .
\end{aligned}
$$

The lower approximation $\operatorname{Apr}(A)$ is the union of all the equivalence classes which are subsets of $A$, and the upper approximation $\overline{A p r}(A)$ is the union of all the equivalence classes which have a non-empty intersection with $A$. The pair $(\operatorname{Apr}(A), \overline{A p r}(A))$ is called

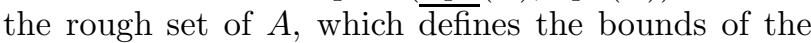
set $A$.

The following list summarizes properties of the rough-set approximations [8]. For any subsets $A, B \subseteq$ $U$ :

$$
\begin{aligned}
& \underline{A p r}(A) \subseteq \overline{A p r}(A), \\
& \overline{A p r}(A \cap B)=\overline{A p r}(A) \cap \underline{A p r}(B), \\
& \overline{A p r}(A \cap B) \subseteq \overline{\overline{A p r}}(A) \cap \overline{\overline{A p r}}(B), \\
& \underline{A p r}(A \cup B) \supseteq \underline{A p r}(A) \cup \underline{A p r}(B), \\
& \overline{A p r}(A \cup B)=\overline{\overline{A p r}}(A) \cup \overline{\overline{A p r}}(B), \\
& \underline{A p r}(A-B)=\underline{A p r}(A)-\overline{A p r}(B),
\end{aligned}
$$




$$
\begin{aligned}
& \overline{A p r}(A-B) \subseteq \overline{A p r}(A)-\underline{A p r}(B), \\
& A \subseteq B \Longrightarrow \underline{A p r}(A) \subseteq \underline{A p r}(B), \\
& A \subseteq B \Longrightarrow \overline{\overline{A p r}}(A) \subseteq \overline{A p r}(B) .
\end{aligned}
$$

According to property (R0), $\operatorname{Apr}(A) \subseteq \overline{\operatorname{Apr}}(A)$, a rough set $(\operatorname{Apr}(A), \overline{\operatorname{Apr}}(A))$ forms an interval set $[\operatorname{Apr}(A), \overline{\operatorname{Apr}(A)}]$. Moreover, properties (R1)-(R3) conform to the definition of operations on interval sets. For instance, suppose $[\operatorname{Apr}(A), \overline{A p r}(A)]$ and $[\underline{A p r}(B), \overline{A p r}(B)]$ are the interval sets generated by the rough sets of $A$ and $B$, namely, $A$ lies in $[\operatorname{Apr}(A), \overline{A p r}(A)]$ and $B$ lies in $[\operatorname{Apr}(B), \overline{A p r}(B)]$.

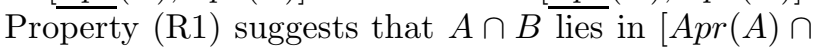
$\operatorname{Apr}(B), \overline{A p r}(A) \cap \overline{A p r}(B)]$, which is consistent with the interval-set intersection. Similarly, Property (R4) supports the definition of interval-set inclusion.

From the above discussion, it is evident that the rough-set model provides a method for constructing an interval set and justifies the use of interval-set operations. Although the notion of interval sets is closely related to rough sets, they are not the same concept. It is true that the rough-set model provides one way to construct interval sets. It is equally important to recognize that the rough-set model is not the only way to achieve this goal. For example, Bundy [2] used interval sets to represent incidences of various propositions in incidence calculus. Thus, rough-set model might be interpreted as a special case of interval-set algebra. In fact, as shown in the next section, interval sets may also be constructed using the fuzzy-set model.

\subsection{Fuzzy sets}

An ordinary set of objects can be defined in terms of a characteristic (membership) function that maps the objects to the two-point set $\{0,1\}$. By extending this notion, Zadeh [11] introduced the concept of fuzzy sets, in which membership functions are defined in the unit interval $[0,1]$. That is, a fuzzy set $\tilde{A}$ is defined by a membership function $\mu_{\tilde{A}}: U \longrightarrow[0,1]$.

Many definitions of fuzzy-set intersection and union have been proposed. The following discussion adopts the classical definition proposed by Zadeh [11], namely, for all $a \in U$,

$$
\begin{aligned}
& \mu_{\tilde{A} \otimes \tilde{B}}(a)=\min \left(\mu_{\tilde{A}}(a), \mu_{\tilde{B}}(a)\right), \\
& \mu_{\tilde{A} \oplus \tilde{B}}(a)=\max \left(\mu_{\tilde{A}}(a), \mu_{\tilde{B}}(a)\right),
\end{aligned}
$$

where $\otimes$ and $\oplus$ denote the fuzzy-set intersection and union, respectively. A fuzzy set $\tilde{A}$ is a subset of a fuzzy set $\tilde{B}$, written $\tilde{A} \subseteq \tilde{B}$, if $\mu_{\tilde{A}}(a) \leq \mu_{\tilde{B}}(a)$ for all $a \in U$.
Given a fuzzy set $\tilde{A}$, the core and support of $\tilde{A}$ are defined by:

$$
\begin{aligned}
& \tilde{A}_{c}=\left\{a \in U \mid \mu_{\tilde{A}}(a)=1\right\}, \\
& \tilde{A}_{s}=\left\{a \in U \mid \mu_{\tilde{A}}(a)>0\right\},
\end{aligned}
$$

which provide useful qualitative information about a fuzzy set. According to this definition, we have $\tilde{A}_{c}, \tilde{A}_{s} \in 2^{U}$ and $\tilde{A}_{c} \subseteq \tilde{A}_{s}$. Thus, $\left[\tilde{A}_{c}, \tilde{A}_{s}\right]$ is an interval set. The cores and supports of fuzzy sets $\tilde{A} \otimes \tilde{B}$ and $\tilde{A} \oplus \tilde{B}$ may be constructed as follows:

$$
\begin{array}{llrl}
(\tilde{A} \otimes \tilde{B})_{c} & =\tilde{A}_{c} \cap \tilde{B}_{c}, & & (\tilde{A} \otimes \tilde{B})_{s}=\tilde{A}_{s} \cap \tilde{B}_{s}, \\
(\tilde{A} \oplus \tilde{B})_{c}=\tilde{A}_{c} \cup \tilde{B}_{c}, & & (\tilde{A} \oplus \tilde{B})_{s}=\tilde{A}_{s} \cup \tilde{B}_{s} .
\end{array}
$$

These definitions are consistent with the interval-set operations in the interval-set algebra.

In comparing rough sets and fuzzy sets, Dubois and Prade [3] drew the correspondences between the lower approximation of a rough set and the core of a fuzzy set, and between the upper approximation of a rough set and the support of a fuzzy set. Yao and Wong [10] extended the concepts of core and support, using the notion of $\alpha$-cut. Given a real number $\alpha \in(0,1]$, the strong $\alpha$-cut or $\alpha$-level set of a fuzzy set $\tilde{A}$ is defined by

$$
\tilde{A}_{\bar{\alpha}}=\left\{a \in U \mid \mu_{\tilde{A}}(a) \geq \alpha\right\},
$$

which is a subset of $U$. Similarly, given a real number $\alpha \in[0,1)$, the $\alpha$-cut of a fuzzy set $\tilde{A}$ is defined by

$$
\tilde{A}_{\alpha}=\left\{a \in U \mid \mu_{\tilde{A}}(a)>\alpha\right\},
$$

which is also a subset of $U$. These notations give $\tilde{A}_{c}=$ $\tilde{A}_{\overline{1}}$ and $\tilde{A}_{s}=\tilde{A}_{0}$. For two real numbers $\alpha, \beta \in[0,1]$ with $\alpha \geq \beta$, we may form an interval set $\left[\tilde{A}_{\alpha}, \tilde{A}_{\beta}\right]$ as an approximation of a fuzzy set. Such an approximation of a fuzzy set can be regarded as generalized core and support. For clarity, we only consider interval sets generated by $\alpha$-level sets. Interval sets generated by $\alpha$-cuts or a mixture of $\alpha$-cuts and $\alpha$-level sets may be studied in the same manner.

For two real numbers $\alpha, \beta \in[0,1]$ with $\alpha \geq \beta$, the generalized core and support satisfy the following properties:

$$
\begin{aligned}
& \tilde{A}_{\bar{\alpha}} \subseteq \tilde{A}_{\bar{\beta}}, \\
& (\tilde{A} \otimes \tilde{B})_{\bar{\alpha}}=\tilde{A}_{\bar{\alpha}} \cap \tilde{B}_{\bar{\alpha}}, \\
& (\tilde{A} \oplus \tilde{B})_{\bar{\alpha}}=\tilde{A}_{\bar{\alpha}} \cup \tilde{B}_{\bar{\alpha}}, \\
& \tilde{A} \subseteq \tilde{B} \Longrightarrow \tilde{A}_{\bar{\alpha}} \subseteq \tilde{B}_{\bar{\alpha}} .
\end{aligned}
$$

Obviously, property (F0) suggests that $\left[\tilde{A}_{\bar{\alpha}}, \tilde{A}_{\bar{\beta}}\right]$ is an interval set which approximates a fuzzy set $\tilde{A}$. Properties (F1) and (F2) are consistent with the definition 
of interval-set operations. If $\left[\tilde{A}_{\bar{\alpha}}, \tilde{A}_{\bar{\beta}}\right]$ is an approximation of $\tilde{A}$ and $\left[\tilde{B}_{\bar{\alpha}}, \tilde{B}_{\bar{\beta}}\right]$ an approximation of $\tilde{B}$, then the interval set defined by

$$
\left[\tilde{A}_{\bar{\alpha}}, \tilde{A}_{\bar{\beta}}\right] \sqcap\left[\tilde{B}_{\bar{\alpha}}, \tilde{B}_{\bar{\beta}}\right]=\left[\tilde{A}_{\bar{\alpha}} \cap \tilde{B}_{\bar{\alpha}}, \tilde{A}_{\bar{\beta}} \cap \tilde{B}_{\bar{\beta}}\right]
$$

is an approximation of fuzzy set $\tilde{A} \otimes \tilde{B}$. A similar observation is also true for the interval-set union operation. Thus, the approximation of fuzzy sets by interval sets also supports the proposed interval-set algebra.

\section{Conclusion}

This paper proposes that an interval-set algebra is a complement to existing interval analysis methods. The concept of interval sets represents a new kind of sets, represented by a pair of sets known as lower and upper bounds. The introduction of this new notion comes naturally from our inability to characterize a set precisely. The basic properties of interval sets are examined, and operations on interval sets are defined in a manner parallel to that used in defining operations on interval numbers. Those operations are justified by the physical meaning of interval sets. More support to the proposed operations is also provided by the connections between interval sets, rough sets and fuzzy sets. In addition, interval sets may be constructed in both rough-set and fuzzy-set models.

The present study immediately suggests two research topics. The basic operations on interval sets provide necessary tools for reasoning with interval sets. It might be useful to pursue research along this line. The notion of interval numbers provides a useful tool for representing quantitative information, whereas the notion of interval sets for qualitative information. It will be interesting to examine how to combine these two methods in uncertainty management.

\section{Acknowledgements}

The author is indebted to NSERC Canada for supporting this study, and to Xining Li for his valuable comments on this paper.

\section{References}

[1] Alefeld, G. and Herzberger, J. Introduction to Interval Computations, Academic Press: New York, 1983.

[2] Bundy, A. Incidence calculus: a mechanism for probabilistic reasoning. Journal of Automated Reasoning, 1, 263-283, 1985.

[3] Dubois, D. and Prade, H. Rough fuzzy sets and fuzzy rough sets. International Journal of General Systems, 17, 191-209, 1990.

[4] Farinas del Cerro, L. and Prade, H. Rough sets, twofold fuzzy sets and modal logic - fuzziness in indiscernibility and partial information. In: Di Nola A. and Ventre A.G.S. (Eds.), The Mathematics of Fuzzy Systems, 103-120, Verlag TUV Rheinland: Köln, 1986.

[5] Moore, R.E. Interval Analysis, Englewood Cliffs, New Jersey: Prentice-Hall, 1966.

[6] Negoiţă, C.V. and Ralescu, D.A. Applications of Fuzzy Sets to Systems Analysis, Basel: Birkhäuser Verlag, 1975.

[7] Parsons, S. Qualitative and semiqualitative methods for integrating uncertainty handling formalisms. Technical Report, Department of Electronic Engineering, Queen Mary and Westfield College, London, UK, 1991.

[8] Pawlak, Z. Rough sets. International Journal of Computer and Information Sciences, 11, 341356, 1982.

[9] Shafer, G. Perspectives on the theory and practice of belief functions. International Journal of Approximate Reasoning, 4, 323-362, 1990.

[10] Yao, Y.Y. and Wong, S.K.M. A decision theoretic framework for approximating concepts. International Journal of Man-machine Studies, 37, 793-809, 1992..

[11] Zadeh, L.A. Fuzzy sets. Information and Control, 8, 338-353, 1965. 